\title{
Nonlinear thermal radiation in flow induced by a slendering surface accounting thermophoresis and Brownian diffusion
}

\author{
M. Waqas ${ }^{1, a}$, M. Ijaz Khan ${ }^{1, b}$, T. Hayat ${ }^{1,2}$, A. Alsaedi ${ }^{2}$, and M. Imran Khan ${ }^{3, c}$ \\ ${ }^{1}$ Department of Mathematics, Quaid-I-Azam University 45320 Islamabad 44000, Pakistan \\ 2 Nonlinear Analysis and Applied Mathematics (NAAM) Research Group, Department of Mathematics, Faculty of Science, \\ King Abdulaziz University, P. O. Box 80257, Jeddah 21589, Saudi Arabia \\ 3 Institute of Petroleum Engineering, Heriot Watt University, Edinburgh, UK
}

Received: 24 March 2017 / Revised: 12 May 2017

Published online: 28 June 2017

(C) The Author(s) 2017. This article is published with open access at Springerlink.com

\begin{abstract}
Our attention here in this research is on scrutinizing the nonlinear convection characteristics in a flow induced by a slendering surface. Flow expression is developed through electrically conducting Williamson nanomaterial. Nonlinear forms of stretching and free stream velocities are imposed. Consideration of nonlinear thermal radiation, non-uniform heat generation/absorption, Joule and convective heating aspects describe the phenomenon of heat transfer. The zero-mass condition for concentration is also considered. The compatible transformations produce strong nonlinear differential systems. The problems are computed analytically utilizing the bvp4c procedure. Heat transfer rate and drag force are also explained for various physical variables. Our analysis reveals that the heat transfer rate augments via larger radiation parameter and Biot number. Moreover, larger Brownian motion and thermophoresis parameters have opposite characteristics on concentration field. For the verification of the present findings, the results of the presented analysis have been compared with the available works in particular situations and reasonable agreement is noted.
\end{abstract}

\section{Introduction}

The flow investigation in the region of boundary layer via a stretchable sheet is a fascinating research field because of its significance in several utilizations in engineering systems and industries, like wire drawing, glass fiber production, hot rolling, metal spinning, contraction process of metallic plates and aerodynamic extrusion of plastic sheets. In view of such applications, Sakiadis [1] initially modeled the problem of boundary layer approximations by stretchable surfaces. Afterwards, several attempts regarding stretchable flow have been reported (see [2-6]). Besides this, when the temperature difference between the surface and ambient fluid is significantly large, the nonlinear density temperature (NDT) variations influence the flow and heat transfer distributions. Therefore in the buoyancy force expression, nonlinear density temperature variations cannot be neglected. Information related to such phenomenon can be seen in refs. [7-12].

Undoubtedly, energy generation is the most important issue in industrial requirements. Thus scientists are focused to improve heat transport rate of systems which comprise chemical plants, power stations, air conditioning and petrochemical industry. In this regard, several techniques were proposed but are not appropriate because of less thermal conductivity. Consequently thermal scientists established energy materials known as nanomaterials comprising nanoscale particles [13]. The tiny-scale particles in a base fluid are termed as nanofluid. A nanofluid is an improved type of fluid comprising nanometer-sized particles (diameter $<100 \mathrm{~nm}$ ) or fibers suspended in the ordinary fluid. Such feature of high thermal conductivity has been noticed by Masuda et al. [14]. Choi et al. [15] disclosed that the thermal conductivity of the fluid can be improved up to twice that of the base fluid by the addition of a small quantity $(<1 \%$ by volume) of nanoparticles to common heat transport liquids. Nanoliquids have a wide range of applications in microelectronics, microfluidics, solid-state lighting, transportation, medical applications, biomedical industry, detergency,

\footnotetext{
a e-mail: mwaqas@math.qau.edu.pk

b e-mail: mikhan@math.qau.edu.pk

c e-mail: mk42@hw.ac.uk (corresponding author)
} 


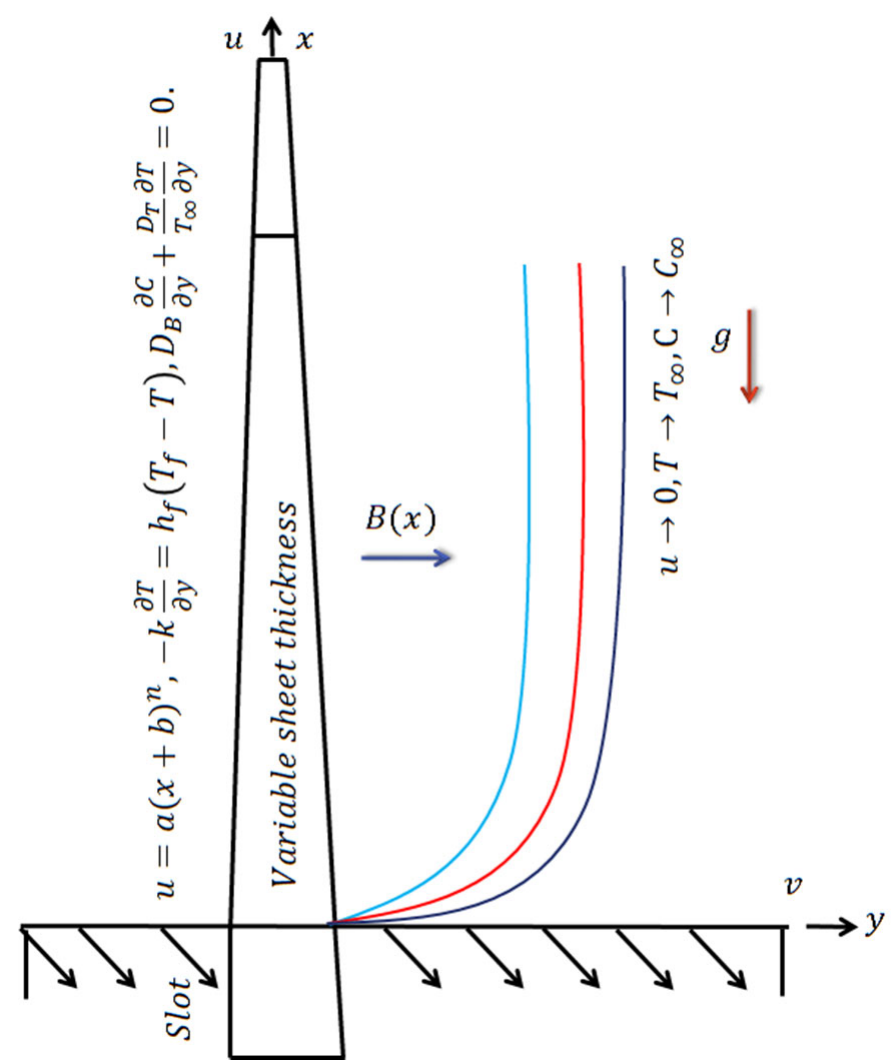

Fig. 1. Geometry of flow problem.

power generation, etc. Besides this, the interaction of magnetic field with nanoliquids has numerous potential applications and may be utilized to deal with problems like chilling of nuclear reactors by liquid sodium and induction flow meter, which relies on the potential difference of the fluid in the direction normal to the motion and to the magnetic field. A number of articles on magneto nanofluids are available in the literature and some are given in refs. [16-25].

The consideration of the nonlinear thermal radiative fluid flow by a stretchable surface is an area of potential interest for researchers due to its demands in several engineering and physical processes. Applicable areas of such processes are solar-power technology, propulsion devices, nuclear plants, combustion chambers, aircraft and chemical processes at high operating temperatures. Especially the thermal radiation aspect plays an important role in regulating the heat transport process in the polymer processing industry. Keeping this in mind, the effect of nonlinear thermal radiation on the Sakiadis flow were firstly reported by Pantokratoras and Fang [26]. Cortell [27] addressed the importance of thermal radiation effect in the stretchable flow of a viscous liquid. Khan et al. [28] developed the series solutions to analyze the nonlinear thermal radiation effect on the Burgers nanofluid flow with convective heating and heat generation/absorption. Hayat et al. [29] investigated the simultaneous characteristics of nonlinear convection and magnetohydrodynamics (MHD) in the Walter-B nanofluid flow induced by a nonlinear stretchable surface in the presence of nonlinear thermal radiation and convective heating. Nonlinear convection and nonlinear thermal radiation impacts on the Maxwell nanofluid by convectively heated stretched sheet are reported by Mahanthesh et al. [30].

The intention here is to investigate the feature of convective heating and zero-mass flux conditions in nonlinear convective flow of a magnetic Williamson liquid. The flow is generated due to a slendering surface. The phenomenon of nanoparticles occurs due to consideration of thermophoretic and Brownian motion. Nonlinear thermal radiation, heat generation/absorption and Joule heating are incorporated in the energy transport expression. The governed mathematical expressions are calculated through a numerical algorithm [31-34]. The significance of arising non-dimensional variables is discussed through graphs and tables.

\section{Mathematical formulation}

Let us scrutinize the two-dimensional (2D) nonlinear convective flow of a magneto Williamson nanomaterial towards a slendering surface at $y=A_{1}(x+b)^{\frac{1-n}{2}}$. The considered nanoliquid model includes the salient characteristics of thermophoresis and Brownian motion. The nanofluid is conducted electrically through the non-uniform magnetic field $\left(B(x)=B_{0}(x+b)^{\frac{n-1}{2}}\right.$ ) operated perpendicularly to the flow trend (see fig. 1). The supposition of a small magnetic Reynolds number leads to negligible characteristics of electric field. Besides this, the nonlinear thermal radiation, heat 
generation/absorption, Joule and convective heating effects are also considered in the heat transfer process. In view of these assumptions the governing expressions for the present situation are $[29,35]$ :

$$
\begin{aligned}
\frac{\partial u}{\partial x}+\frac{\partial v}{\partial y}= & 0 \\
u \frac{\partial u}{\partial x}+v \frac{\partial u}{\partial y}= & \nu \frac{\partial^{2} u}{\partial y^{2}}+\sqrt{2} \nu \Gamma\left(\frac{\partial u}{\partial y}\right) \frac{\partial^{2} u}{\partial y^{2}}-\frac{\sigma B^{2}(x)}{\rho_{f}} u \\
& +g\left\{\Lambda_{1}\left(T-T_{\infty}\right)+\Lambda_{2}\left(T-T_{\infty}\right)^{2}\right\}+g\left\{\Lambda_{3}\left(C-C_{\infty}\right)+\Lambda_{4}\left(C-C_{\infty}\right)^{2}\right\}, \\
u \frac{\partial T}{\partial x}+v \frac{\partial T}{\partial y}= & \alpha^{*} \frac{\partial^{2} T}{\partial y^{2}}+\frac{\sigma B^{2}(x)}{(\rho c)_{f}} u^{2}+\tau\left(D_{B} \frac{\partial C}{\partial y} \frac{\partial T}{\partial y}+\frac{D_{T}}{T_{\infty}}\left(\frac{\partial T}{\partial y}\right)^{2}\right)+\frac{1}{(\rho c)_{f}} \frac{16 \sigma^{*}}{3 k^{*}} \frac{\partial}{\partial y}\left(T^{3} \frac{\partial T}{\partial y}\right)+\frac{Q(x)}{(\rho c)_{f}}\left(T-T_{\infty}\right), \\
u \frac{\partial C}{\partial x}+v \frac{\partial C}{\partial y}= & D_{B} \frac{\partial^{2} C}{\partial y^{2}}+\frac{D_{T}}{T_{\infty}} \frac{\partial^{2} T}{\partial y^{2}},
\end{aligned}
$$

for conditions $[29]$

$$
\begin{aligned}
& u=u_{w}(x)=a(x+b)^{n}, \quad v=0, \quad-k \frac{\partial T}{\partial y}=h_{f}\left(T_{f}-T\right), \\
& D_{B} \frac{\partial C}{\partial y}+\frac{D_{T}}{T_{\infty}} \frac{\partial T}{\partial y}=0 \quad \text { at } \quad y=A_{1}(x+b)^{\frac{1-n}{2}}, \\
& u \longrightarrow 0, \quad T \longrightarrow T_{\infty}, \quad C \longrightarrow C_{\infty} \quad \text { as } \quad y \longrightarrow \infty,
\end{aligned}
$$

in which $(u, v)$ are the velocity elements along the $(x, y)$ axes, respectively, $\nu=\left(\frac{\mu}{\rho_{f}}\right)$ the kinematic viscosity with $\mu$ the fluid kinematic viscosity and $\rho_{f}$ the base fluid density, $\alpha^{*}=\frac{k}{(\rho c)_{f}}$ the fluid thermal conductivity, $\Gamma$ the material parameter of Williamson fluid, $B_{0}$ the magnetic field strength, $(T, C)$ the fluid (temperature, concentration), $T_{f}$ the convective fluid temperature, $\left(T_{\infty}, C_{\infty}\right)$ the ambient (temperature, concentration), $k$ the fluid thermal conductivity, $\tau=\frac{(\rho c)_{p}}{(\rho c)_{f}}$ the heat capacity ratio, $(\rho c)_{f}$ the heat capacity of base fluid, $(\rho c)_{p}$ the effective heat capacity of nanoparticles, $\left(D_{T}, D_{B}\right)$ the (thermophoretic, Brownian) diffusion coefficients, $\sigma^{*}$ the Stefan-Boltzmann constant, $k^{*}$ the mean absorption coefficient, $Q(x)=Q_{0}(x+b)^{n-1}$ the non-uniform heat generation/absorption, $\left(\Lambda_{1}, \Lambda_{2}\right)$ the (linear, nonlinear) thermal expansion coefficients, $\left(\Lambda_{3}, \Lambda_{4}\right)$ the (linear, nonlinear) concentration expansion coefficients, $u_{w}(x)$ the stretching velocity, $n$ the power index and $h_{f}=h(x+b)^{\frac{n-1}{2}}$ the non-uniform heat transfer coefficient and $(a, b)$ the dimensional constants.

Letting [29]

$$
\begin{aligned}
\psi & =\sqrt{\left(\frac{2}{n+1}\right) a \nu(x+b)^{n+1}} F(\xi), \quad \xi=y \sqrt{\left(\frac{n+1}{2}\right)\left(\frac{a}{\nu}\right)(x+b)^{n-1}}, \\
u & =a(x+b)^{n} F^{\prime}(\xi), \quad v=-\sqrt{\left(\frac{n+1}{2}\right) a \nu(x+b)^{n-1}}\left(F(\xi)+\xi \frac{n-1}{n+1} F^{\prime}(\xi)\right), \\
\Theta(\xi) & =\frac{T-T_{\infty}}{T_{f}-T_{\infty}}, \quad \Phi(\xi)=\frac{C-C_{\infty}}{C_{\infty}},
\end{aligned}
$$

eq. (1) is trivially satisfied and other equations are reduced to

$$
\begin{aligned}
& F^{\prime \prime \prime}-\frac{2 n}{n+1} F^{2}+F F^{\prime \prime}+W e \sqrt{\frac{n+1}{2}} F^{\prime \prime} F^{\prime \prime \prime}-\frac{2}{n+1} M^{2} F^{\prime}+\frac{2}{n+1} \lambda\left[\left(1+\beta_{t} \Theta\right) \Theta+N\left(1+\beta_{c} \Phi\right) \Phi\right]=0 \\
& {\left[1+R\left\{1+\left(\Theta_{f}-1\right) \Theta\right\}^{3} \Theta^{\prime}\right]^{\prime}+\operatorname{Pr}\left(F \Theta^{\prime}+N b \Theta^{\prime} \Phi^{\prime}+N t \Theta^{\prime 2}+\frac{2}{n+1} \delta \Theta+E c M^{2} F^{2}\right)=0} \\
& \Phi^{\prime \prime}+S c F \Phi^{\prime}+\frac{N t}{N b} \Theta^{\prime \prime}=0 \\
& F(\alpha)=\alpha\left(\frac{1-n}{1+n}\right), \quad F^{\prime}(\alpha)=1, \quad \Theta^{\prime}(\alpha)=-\gamma(1-\Theta(\alpha)) \\
& N b \Phi^{\prime}(\alpha)+N t \Theta^{\prime}(\alpha)=0 \quad \text { at } \alpha=A_{1} \sqrt{\frac{n+1}{2}} \frac{a}{\nu} \\
& F^{\prime}(\alpha) \rightarrow 0, \quad \Theta(\alpha) \rightarrow 0, \quad \Phi(\alpha) \rightarrow 1 \quad \text { as } \alpha \rightarrow \infty
\end{aligned}
$$


Now, setting $F(\xi)=f(\xi-\alpha)=f(\eta), \Theta(\xi)=\theta(\xi-\alpha)=\theta(\eta)$ and $\Phi(\xi)=\phi(\xi-\alpha)=\phi(\eta)$ yields eqs. (8)-(11) in the following forms:

$$
\begin{aligned}
& f^{\prime \prime \prime}-\frac{2 n}{n+1} f^{2}+f f^{\prime \prime}+W e \sqrt{\frac{n+1}{2}} f^{\prime \prime} f^{\prime \prime \prime}-\frac{2}{n+1} M^{2} f^{\prime}+\frac{2}{n+1} \lambda\left[\left(1+\beta_{t} \theta\right) \theta+N\left(1+\beta_{c} \theta\right) \theta\right]=0 \\
& {\left[1+R\left\{1+\left(\theta_{f}-1\right) \theta\right\}^{3} \theta^{\prime}\right]^{\prime}+\operatorname{Pr}\left(f \theta^{\prime}+N b \theta^{\prime} \phi^{\prime}+N t \theta^{\prime 2}+\frac{2}{n+1} \delta \theta+E c M^{2} f^{\prime 2}\right)=0,} \\
& \phi^{\prime \prime}+S c f \phi^{\prime}+\frac{N t}{N b} \theta^{\prime \prime}=0 \\
& f(0)=\alpha\left(\frac{1-n}{1+n}\right), \quad f^{\prime}(0)=1, \quad \theta^{\prime}(0)=-\gamma(1-\theta(0)) \\
& N_{b} \phi^{\prime}(0)+N_{t} \theta^{\prime}(0)=0, \\
& f^{\prime}(0) \rightarrow 0, \quad \theta(0) \rightarrow 0, \quad \phi(0) \rightarrow 1
\end{aligned}
$$

Here the Weissenberg number $(W e)$, Hartman number $(M)$, thermal buoyancy parameter $(\lambda)$, Grashof number in terms of temperature $\left(G r_{x}\right)$, local Reynolds number $\left(\operatorname{Re}_{x}\right)$, nonlinear thermal convection parameter $\left(\beta_{t}\right)$, nonlinear solutal convection parameter $\left(\beta_{c}\right)$, ratio of concentration to thermal buoyancy forces $(N)$, Grashof number in terms of concentration $\left(G r_{x}^{*}\right)$, temperature ratio parameter $\left(\theta_{f}\right)$, radiation parameter $(R)$, Prandtl number $(\operatorname{Pr})$, heat generation $(\delta>0)$, heat absorption $(\delta<0)$, thermophoretic parameter $\left(N_{t}\right)$, Eckert number $(E c)$, Brownian motion parameter $\left(N_{b}\right)$, Biot number $(\gamma)$, Schmidt number $(S c)$ and wall thickness parameter $(\alpha)$ are stated as follows:

$$
\begin{aligned}
W e & =\Gamma\left(\frac{a^{3}(x+b)^{3 n-1}}{\nu}\right)^{1 / 2}, \quad M^{2}=\frac{\sigma B_{0}^{2}}{\rho_{f} a}, \quad \lambda=\frac{G r_{x}}{\operatorname{Re}_{x}^{2}}, \quad G r_{x}=\frac{g \Lambda_{1}\left(T_{f}-T_{\infty}\right)(x+b)^{3}}{\nu^{2}}, \\
\operatorname{Re}_{x} & =\frac{u_{w}}{\nu}(x+b), \quad \beta_{t}=\frac{\Lambda_{2}\left(T_{f}-T_{\infty}\right)}{\Lambda_{1}}, \quad \beta_{c}=\frac{\Lambda_{4} C_{\infty}}{\Lambda_{3}}, \quad N=\frac{G r_{x}^{*}}{G r_{x}}, \quad G r_{x}^{*}=\frac{g \Lambda_{3} C_{\infty}(x+b)^{3}}{\nu^{2}}, \\
\theta_{f} & =\frac{T_{f}}{T_{\infty}}, \quad R=\frac{16 \sigma^{*} T_{\infty}^{3}}{3 k^{*} k}, \quad \operatorname{Pr}=\frac{\nu}{\alpha^{*}}, \quad \delta=\frac{Q_{0}}{(\rho c)_{f} a}, \quad N_{t}=\frac{\tau D_{T}\left(T_{f}-T_{\infty}\right)}{T_{\infty} \nu}, \\
E c & =\frac{u_{w}^{2}}{c_{p}\left(T_{f}-T_{\infty}\right)}, \quad \gamma=\frac{h}{k} \sqrt{\frac{\nu}{\alpha^{*}}}, \quad N_{b}=\frac{\tau D_{B} C_{\infty}}{\nu}, \quad S c=\frac{\nu}{D_{B}}, \quad \alpha=A_{1} \sqrt{\frac{n+1}{2}} \frac{a}{\nu}
\end{aligned}
$$

The surface drag force $\left(C_{f}\right)$ and local Nusselt number $(N u)$ are

$$
C_{f}=\frac{\tau_{w}}{\rho u_{w}^{2}}, \quad N u=\frac{(x+b) q_{w}}{k\left(T_{f}-T_{\infty}\right)} .
$$

The following relations will be satisfied for surface shear stress $\left(\tau_{w}\right)$ and surface heat flux $\left(q_{w}\right)$, i.e.

$$
\begin{aligned}
& \tau_{w}=\left.\mu\left(\frac{\partial u}{\partial y}+\frac{\Gamma}{\sqrt{2}}\left(\frac{\partial u}{\partial y}\right)^{2}\right)\right|_{y=A_{1}(x+b)^{\frac{1-n}{2}}}, \\
& q_{w}=-\left.k\left(1+\frac{16 \sigma^{*} T^{3}}{3 k k^{*}}\right)\left(\frac{\partial T}{\partial y}\right)\right|_{y=A_{1}(x+b)^{\frac{1-n}{2}}}
\end{aligned}
$$

Replacing eq. (18) in eq. (17) one can obtain

$$
\begin{gathered}
\sqrt{\operatorname{Re}_{x}} C_{f}=\left(\sqrt{\frac{n+1}{2}} f^{\prime \prime}(0)+W e \frac{n+1}{4}\left(f^{\prime \prime}(0)\right)^{2}\right), \\
N u \operatorname{Re}_{x}^{-1 / 2}=-\sqrt{\frac{n+1}{2}}\left(1+R\left\{1+\left(\theta_{f}-1\right) \theta(0)\right\}^{3}\right) \theta^{\prime}(0) .
\end{gathered}
$$

\section{Solution methodology}

\subsection{Computational procedure}

This subsection reports the numeric solutions of developed nonlinear systems (12)-(14) subject to boundary conditions (15) through the bvp4c technique. 
Table 1. Comparative results of surface drag force with [35] through different $M$, when $n=1.0$ and $\lambda=0.0=\alpha=N=\beta_{t}=$ $\beta_{c}=W e$.

\begin{tabular}{lll}
\hline \multicolumn{2}{c}{$-f^{\prime \prime}(0)$} \\
\hline$M$ & Ref. [35] & Present results \\
\hline 0.0 & 1.00000 & 1.00000 \\
0.2 & 1.01980 & 1.01980 \\
0.5 & 1.11803 & 1.11803 \\
0.8 & 1.28063 & 1.28063 \\
1.0 & 1.41421 & 1.41421 \\
1.2 & 1.56205 & 1.56205 \\
1.5 & 1.80303 & 1.80303 \\
\hline
\end{tabular}

Table 2. Surface drag force $\left(C_{f} \operatorname{Re}_{x}^{1 / 2}\right)$ through distinct values of $M, W e, \lambda, \beta_{t}$ and $\alpha$, when $n=0.9, \delta=0.1, N=N_{b}=R=$ $\gamma=E c=0.5, \beta_{t}=0.2, S c=\theta_{f}=1.1, \operatorname{Pr}=1.2$ and $N_{t}=0.3$.

\begin{tabular}{llllll}
\hline$M$ & $W e$ & $\lambda$ & $\beta_{t}$ & $\alpha$ & $C_{f} \operatorname{Re}_{x}^{1 / 2}$ \\
\hline 0.0 & 1.0 & 0.5 & 0.3 & 0.2 & -0.007052794 \\
0.3 & & & & & -0.05752041 \\
0.5 & & & & & -0.2758314 \\
0.3 & 0.0 & & & & -0.8824139 \\
& 0.4 & & & & -0.6697345 \\
& 0.8 & & & & -0.3299715 \\
& 1.0 & 0.6 & & & -0.3015025 \\
& & 0.8 & & & -0.2990457 \\
& & 0.9 & & & -0.2977894 \\
& & 0.5 & 0.2 & & -0.2563464 \\
& & 0.4 & & -0.2388347 \\
& & 0.5 & & -0.1264708 \\
& & & 0.3 & 0.0 & -0.2882989 \\
& & & & 0.1 & -0.2395947 \\
& & & 0.5 & -0.05607216
\end{tabular}

\subsection{Testing the numerical scheme}

The validation of the employed technique with the available published results [36] in a limiting manner is elaborated in this subsection through table 1. It is noticed that our results are in reasonable agreement with [36].

\section{Analysis}

The salient features of distinct physical variables like Weissenberg number $(W e)$, Hartman number $(M)$, thermal buoyancy parameter $(\lambda)$, nonlinear thermal convection parameter $\left(\beta_{t}\right)$, nonlinear solutal convection parameter $\left(\beta_{c}\right)$, ratio of concentration to thermal buoyancy forces $(N)$, temperature ratio parameter $\left(\theta_{f}\right)$, radiation parameter $(R)$, Prandtl number $(\operatorname{Pr})$, heat generation parameter $(\delta>0)$, heat absorption parameter $(\delta<0)$, thermophoretic parameter $\left(N_{t}\right)$, Eckert number $(E c)$, Brownian motion parameter $\left(N_{b}\right)$, Biot number $(\gamma)$, Schmidt number $(S c)$ and wall thickness parameter $(\alpha)$ on the non-dimensional temperature $(\theta)$, nanoparticle concentration $(\phi)$, surface drag force $\left(C_{f} \operatorname{Re}_{x}^{1 / 2}\right)$ and Nusselt number $\left(N u_{x} \operatorname{Re}_{x}^{-1 / 2}\right)$ are illustrated and interpreted in figs. 2-13 and tables 2 and 3 .

Figure 2 displays the nature of the temperature field with $N_{b}$. Here the strong Brownian diffusion leads to an upsurge in the temperature and the associated thickness of the thermal boundary layer. Small elements in the thermophoresis mechanism are dragged aside from the warm surface to the chilly one. Thus a huge amount of nanoparticles will move apart from the warm surface, which increases the liquid temperature. The radiation impact on the temperature $(\theta)$ is exhibited in fig. 3. Clearly the ascending values of the thermal radiation factor $(R)$ enhance the temperature field. 
Table 3. Heat transfer rate $\left(N u \operatorname{Re}_{x}^{-1 / 2}\right)$ through distinct values of $N_{t}, N_{b}, R, \theta_{f}, \gamma, \delta$, Pr and $E c$, when $n=0.9, W e=1.0$, $\lambda=N=E c=0.5, \beta_{t}=M=0.3, \beta_{t}=\alpha=0.2$ and $S c=1.1$.

\begin{tabular}{|c|c|c|c|c|c|c|c|}
\hline$N_{t}$ & $N_{b}$ & $R$ & $\theta_{f}$ & $\gamma$ & $\delta$ & $\operatorname{Pr}$ & $N u \operatorname{Re}_{x}^{-1 / 2}$ \\
\hline 0.2 & 1.0 & 0.5 & 0.3 & 0.2 & 0.1 & 1.2 & 0.201343 \\
\hline 0.4 & & & & & & & 0.207022 \\
\hline 0.5 & & & & & & & 0.210507 \\
\hline \multirow[t]{18}{*}{0.3} & 0.2 & & & & & & 0.21686 \\
\hline & 0.3 & & & & & & 0.211506 \\
\hline & 0.4 & & & & & & 0.206768 \\
\hline & 0.5 & 0.0 & & & & & 0.264135 \\
\hline & & 0.4 & & & & & 0.227062 \\
\hline & & 0.8 & & & & & 0.213529 \\
\hline & & 0.5 & 1.2 & & & & 0.179254 \\
\hline & & & 1.3 & & & & 0.144851 \\
\hline & & & 1.4 & & & & 0.120746 \\
\hline & & & 1.1 & 0.3 & & & 0.170484 \\
\hline & & & & 0.6 & & & 0.220432 \\
\hline & & & & 0.9 & & & 0.232434 \\
\hline & & & & 0.5 & 0.0 & & 0.274422 \\
\hline & & & & & 0.2 & & 0.337643 \\
\hline & & & & & 0.4 & & 0.82374 \\
\hline & & & & & 0.1 & 1.1 & 0.196687 \\
\hline & & & & & & 1.3 & 0.257983 \\
\hline & & & & & & 1.5 & 0.316771 \\
\hline
\end{tabular}

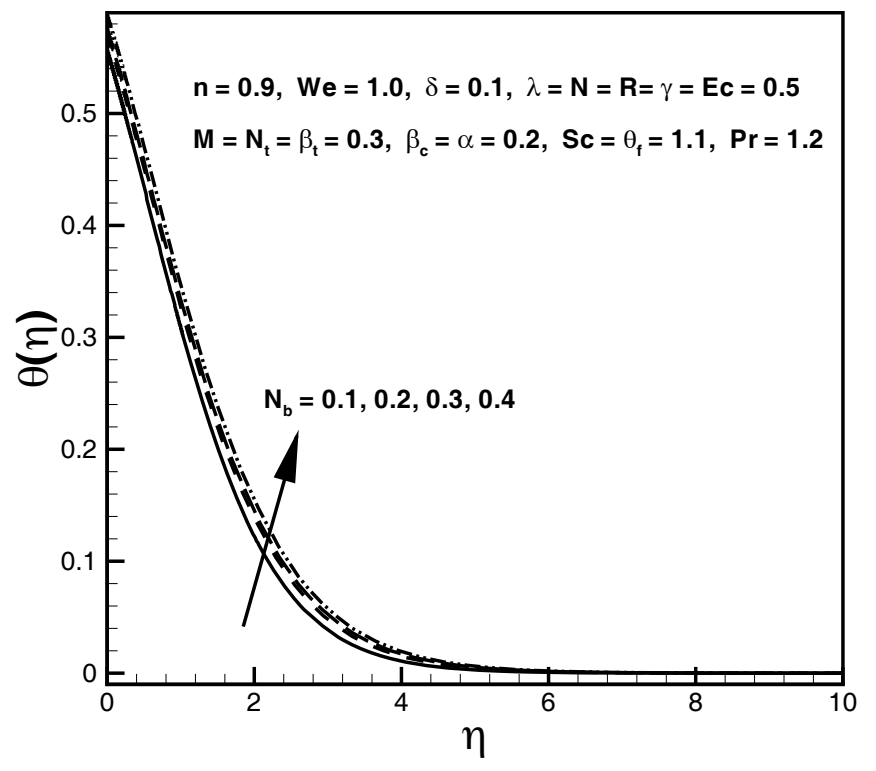

Fig. 2. $\theta$ via $N_{b}$. 


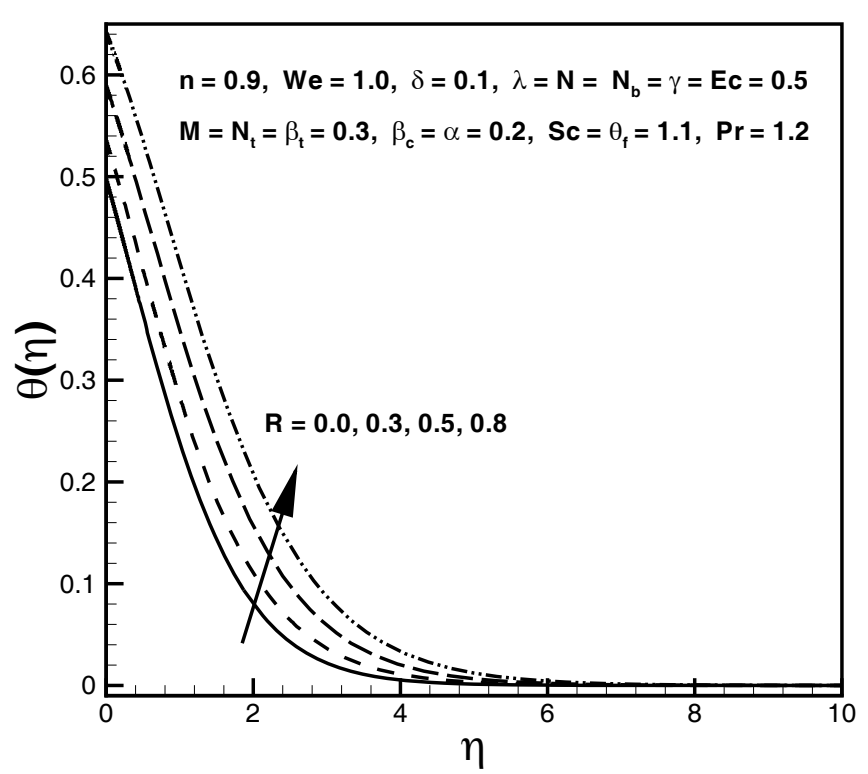

Fig. 3. $\theta$ via $R$.

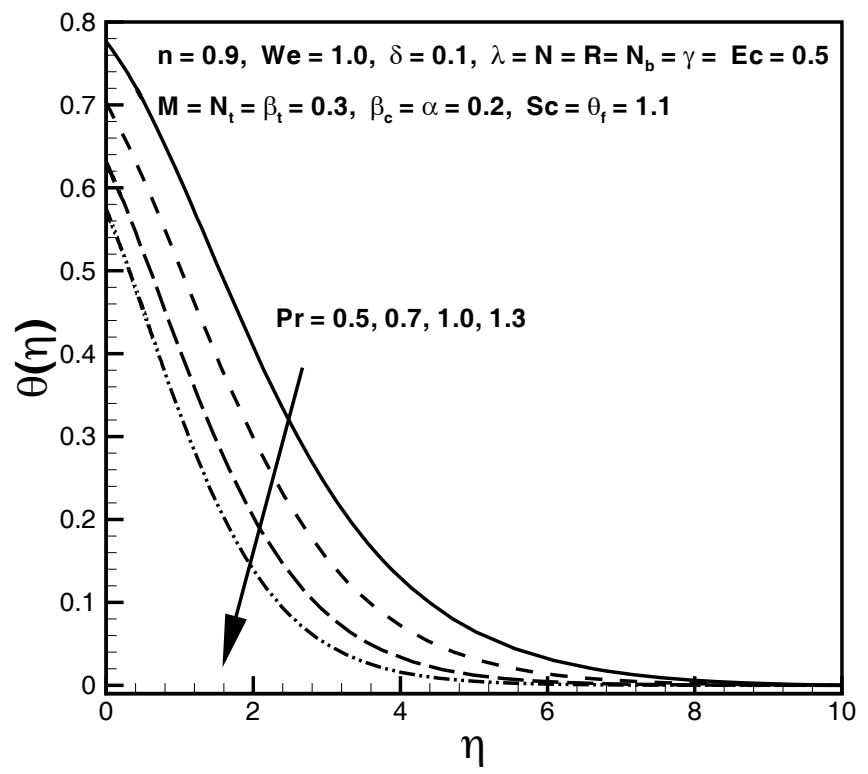

Fig. 4. $\theta$ via $\operatorname{Pr}$.

In fact, radiation is the diffusion of heat energy from one section to another. Thermal radiation yields peripheral heat energy to augment the temperature field. Figure 4 shows the effect of the Prandtl number (Pr) on the temperature $(\theta)$. Since $\operatorname{Pr}$ is contrariwise proportional to the thermal diffusivity, escalating values of Pr yield a weaker thermal diffusivity. Larger Prandtl liquids produce weaker thermal diffusivity and lower Prandtl liquids have stronger thermal diffusivity. Such weaker thermal diffusivity yields a decrease in temperature and its associated boundary layer thickness. The variation of temperature $(\theta)$ for different values of $\theta_{f}$ is plotted in fig. 5 . It is found that the temperature $(\theta)$ and thickness of thermal boundary layer increase through larger $\theta_{f}$. This happens due to the fact that when we increment $\theta_{f}$, then $T_{f}$ upsurges, due to which more heat is transferred to the fluid and so the temperature profile increases. Figure 6 exhibits the effect of $\gamma$ on the temperature $(\theta)$. It is observed that both the temperature and 


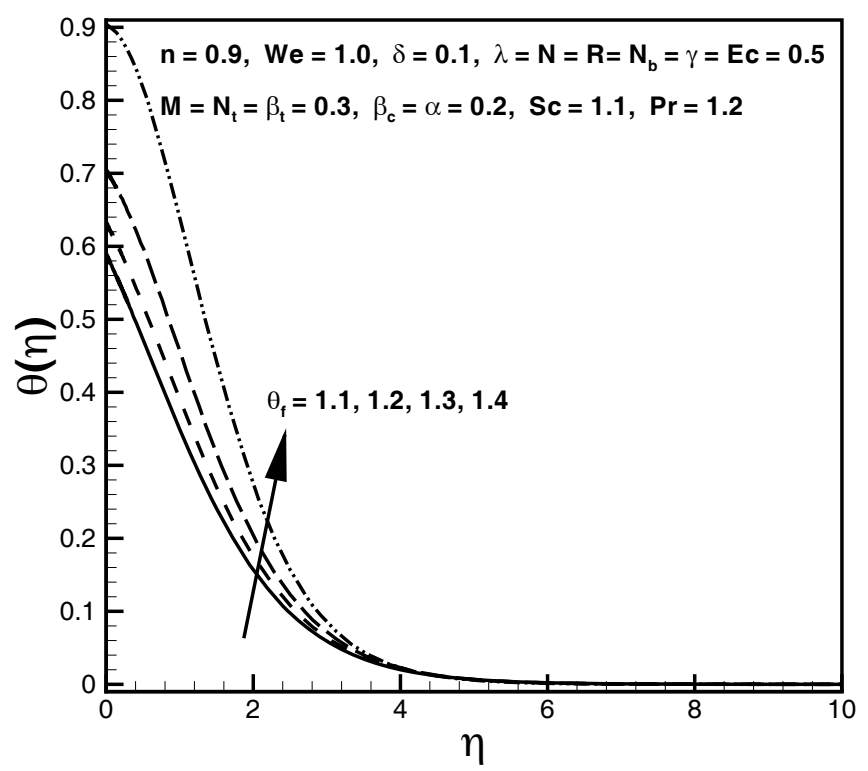

Fig. 5. $\theta$ via $\theta_{f}$.

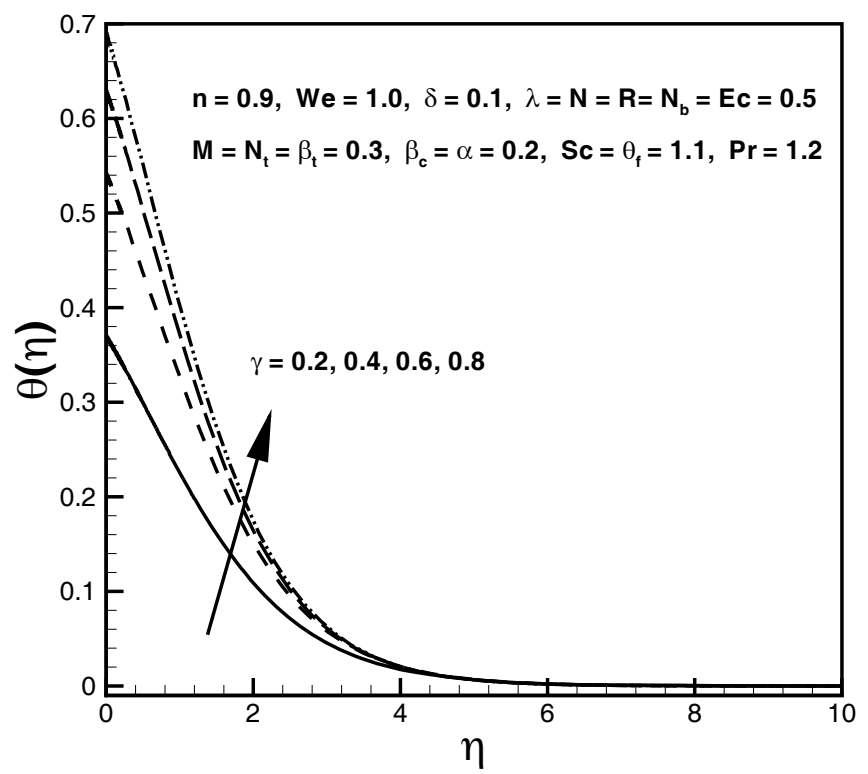

Fig. 6. $\theta$ via $\gamma$.

associated thickness of thermal layer are higher via larger $\gamma$. Physically the heat transfer coefficient is directly related to the Biot number, which is enhanced for larger $\gamma$. This enhancement in the heat transfer coefficient leads to higher temperature. Figures 7 and 8 show the influence of heat source or heat generation $(\delta>0)$ and heat sink or heat absorption $(\delta<0)$ on temperature $(\theta)$. It is observed that the temperature increases throughout the boundary layer region as $(\delta>0)$ increases; however, a reverse scenario is noticed for heat sink or heat generation $(\delta<0)$. This is because the heat generation or heat source parameter $(\delta>0)$ provides more heat into the fluid, which leads to an intensification of the temperature and associated thermal boundary layer. The variation of $E c$ on the temperature $(\theta)$ is plotted in fig. 9. Here the temperature $(\theta)$ and the associated thickness of the thermal layer are increasing functions of $E c$. Physically, an increase in $E c$ means that the heat energy is stored in the fluid due to the frictional 


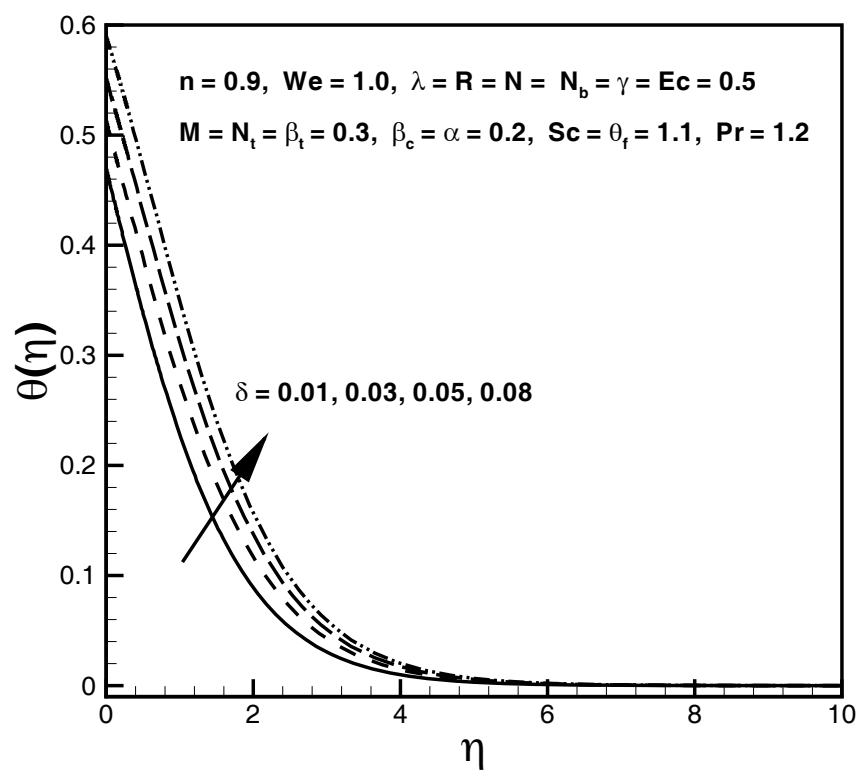

Fig. 7. $\theta$ via $\delta>0$.

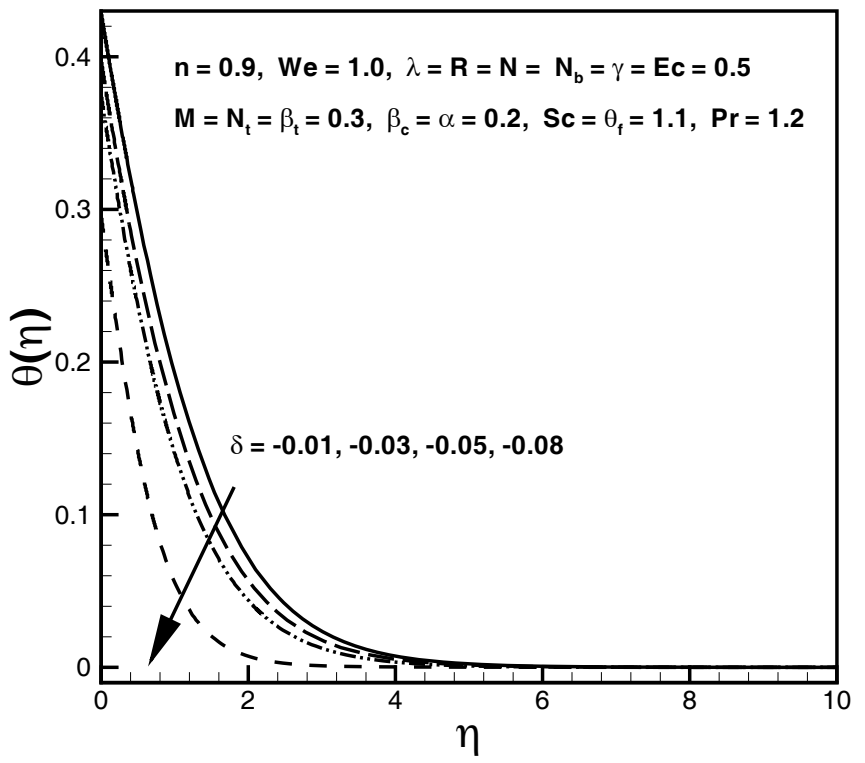

Fig. 8. $\theta$ via $\delta<0$.

or drag forces. As a result, the fluid temperature $(\theta)$ enhances. Figure 10 is drawn to have a good knowledge of the Schmidt number $(S c)$ on nanoparticle concentration $(\phi)$. As the Schmidt number is inversely proportional to the mass diffusion, so a larger $S c$ shows a decay in the nanoparticle concentration $(\phi)$ and its linked thickness of boundary layer. The behavior of $\operatorname{Pr}$ versus nanoparticle concentration $(\phi)$ is addressed through fig. 11. Clearly, a larger Pr leads to a decay in the nanoparticle concentration $(\phi)$ and the corresponding layer thickness. Figure 12 shows the variation of $N_{t}$ on nanoparticle concentration $(\phi)$. Here rising values of $N_{t}$ yield an increment in nanoparticle concentration $(\phi)$. Physically, larger $N_{t}$ creates an additional force and makes nanoparticles pass from the hottest to the coolest region. Therefore nanoparticle concentration $(\phi)$ and its linked boundary layer are higher for $N_{t}$. The variation in the nanoparticle concentration $(\phi)$ as a result of the changes in $N_{b}$ is shown in fig. 13. It is found that increasing values of 


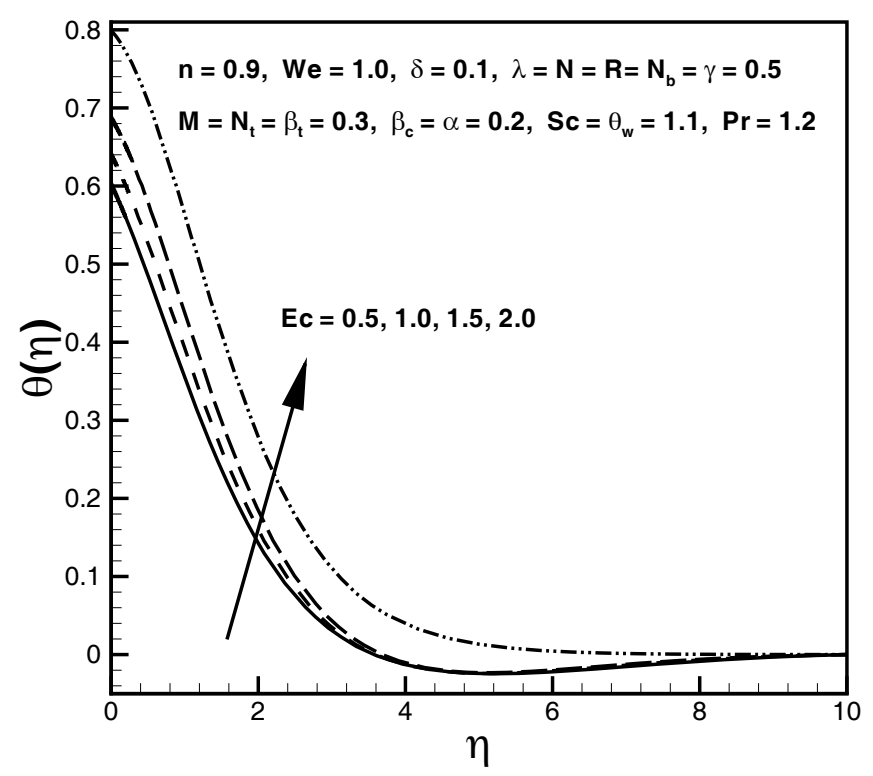

Fig. 9. $\theta$ via $E c$.

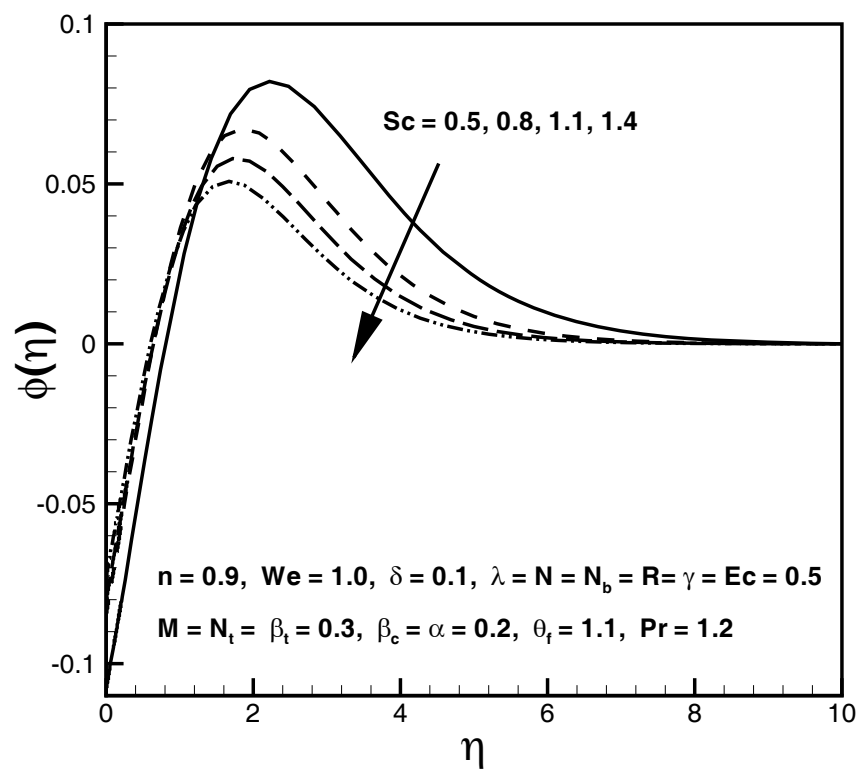

Fig. 10. $\phi$ via $S c$.

$N_{b}$ reduce the nanoparticle concentration $(\phi)$. In fact, higher values of the Brownian motion factor $\left(N_{b}\right)$ help to heat the fluid near the boundary. This leads to a decrease in the nanoparticle concentration $(\phi)$.

Table 2 is built to study the influences of distinct variables, like $H a, W e, \lambda, \beta_{t}$ and $\alpha$, on the surface drag force $\left(C_{f} \sqrt{\mathrm{Re}_{x}}\right)$. Here the surface drag force is an increasing function of $H a$, whereas an opposite trend is noted for $W e$, $\lambda, \beta_{t}$ and $\alpha$. Table 3 elucidates the influence of the Nusselt number for distinct values of $N_{t}, N_{b}, R, \theta_{f}, \gamma, \delta$ and Pr. Increasing values of $N_{t}, \gamma, \delta$ and Pr lead to an enhancement in the Nusselt number, while a reverse behavior is noted for $N_{b}, R$ and $\theta_{f}$. 


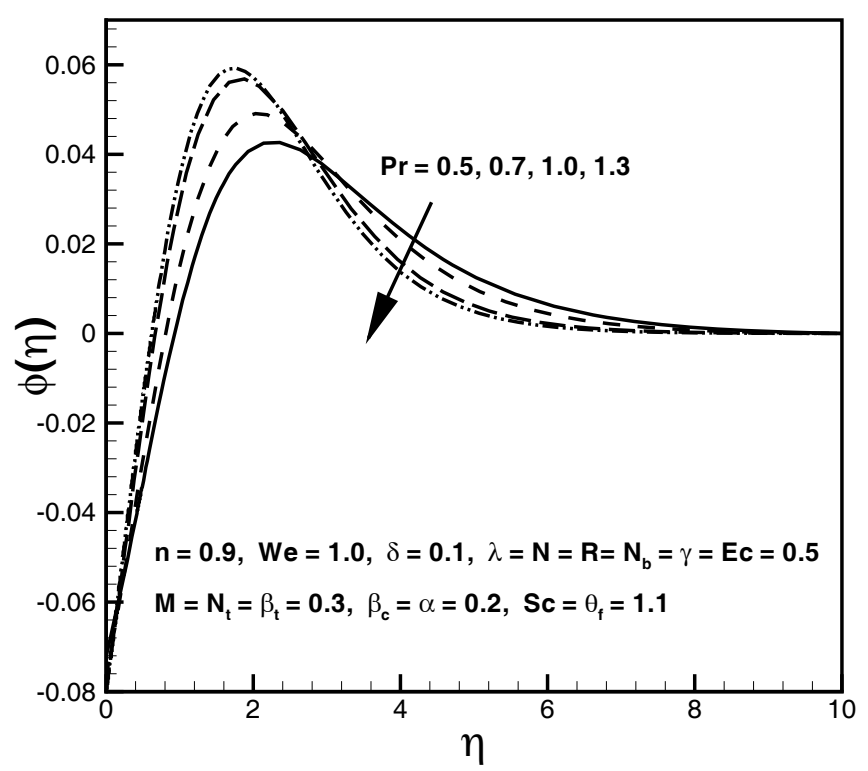

Fig. 11. $\theta$ via Pr.

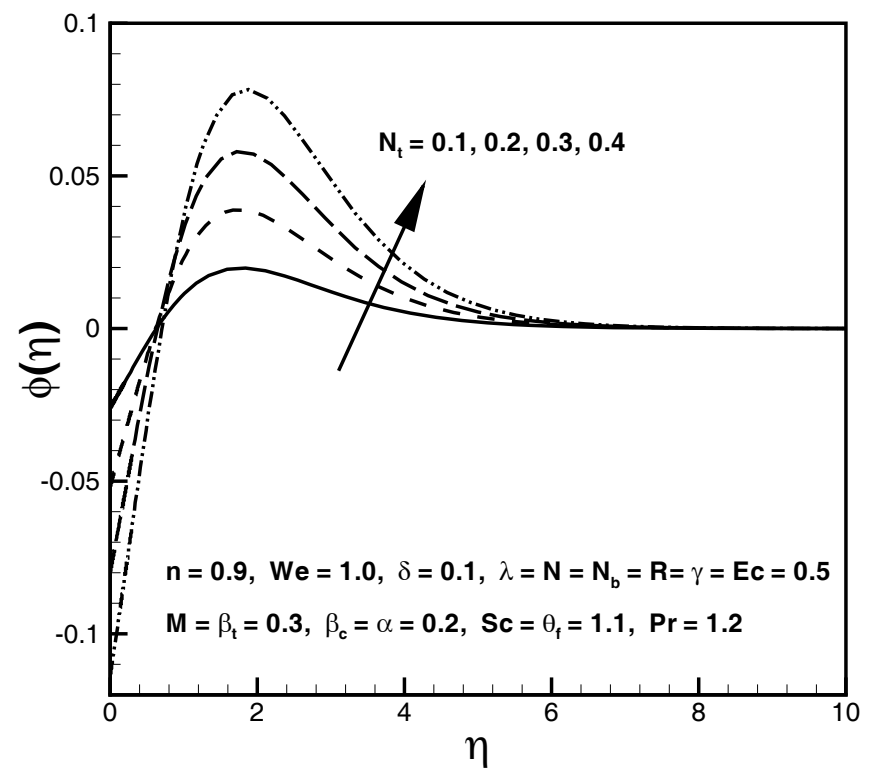

Fig. 12. $\theta$ via $N_{t}$.

\section{Conclusions}

Here we explored the interaction of convective heating in a hydromagnetic Williamson nanoliquid induced due to a slendering surface. The nonlinear thermal radiation, heat generation/absorption and Joule heating are also considered in the heat transport expression. The effect of emerging variables on dimensionless quantities is illustrated in detail. The following key points are drawn from this work:

- A larger Pr corresponds to a decay in temperature and thermal boundary layer thicknesses, whereas a reverse situation is noticed via larger $\gamma$. This reverse behavior is due to weaker thermal diffusivity, in the case of the Prandtl number, and to a larger heat transfer coefficient, in the case of the Biot number. 


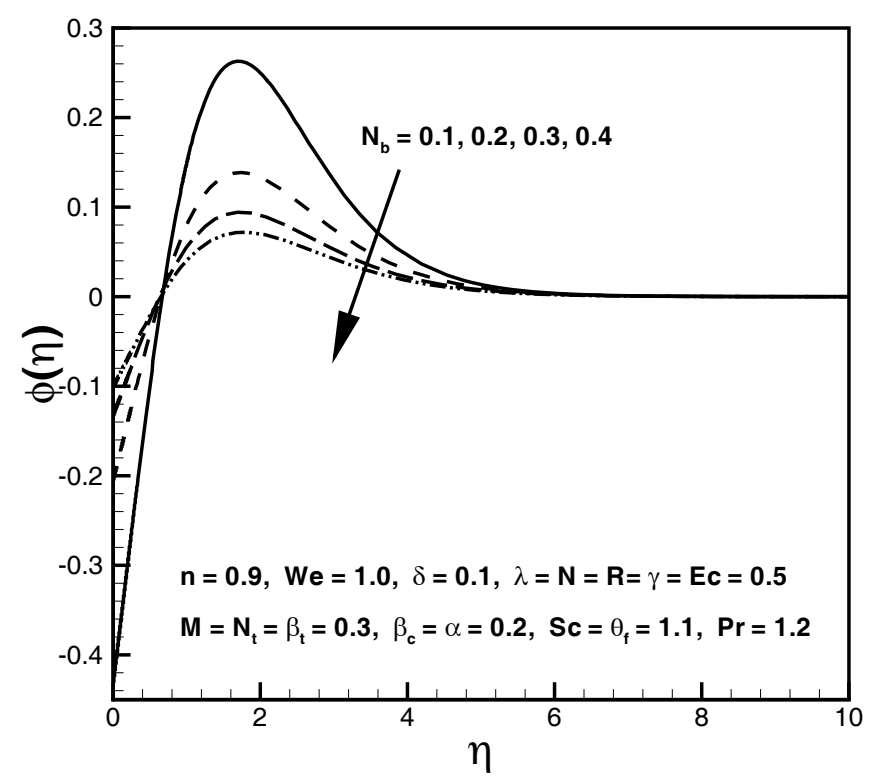

Fig. 13. $\theta$ via $N_{b}$.

- Both temperature distribution and associated thermal layer are enhanced through larger Eckert number $(E c)$, heat generation parameter $(\delta)$, radiation parameter $(R)$ and temperature ratio parameter $\left(\theta_{f}\right)$; however temperature distribution and related thermal layer are lower for heat absorption parameter $(\delta)$.

- The increasing thermophoretic parameter increases the profiles of temperature and concentration.

- Consideration of higher Schmidt number $(S c)$ and Brownian motion factor $\left(N_{b}\right)$ yields lower concentration.

- The values of surface drag force are higher due to the increasing values of the Hartman number $(M)$; however the drag force is reduced for larger Weissenberg number $(W e)$, thermal buoyancy parameter $(\lambda)$, nonlinear thermal convection parameter $\left(\beta_{t}\right)$ and wall thickness parameter $(\alpha)$.

- Larger values of thermophoretic and Brownian motion parameters lead to an enhancement in the temperature profile due to higher thermal conductivity fluid.

- The local Nusselt number enhances for higher values of $N t, \delta$ and $\operatorname{Pr}$.

Open Access This is an open access article distributed under the terms of the Creative Commons Attribution License (http://creativecommons.org/licenses/by/4.0), which permits unrestricted use, distribution, and reproduction in any medium, provided the original work is properly cited.

\section{References}

1. B.C. Sakiadis, AIChE 7, 26 (1961).

2. L. Zheng, J. Niu, X. Zheng, L. Ma, Int. J. Heat Mass Transfer 55, 7577 (2012).

3. M. Khan, W.A. Khan, J. Braz. Soc. Mech. Sci. Eng. 38, 2359 (2016).

4. M. Waqas, T. Hayat, M. Farooq, S.A. Shehzad, A. Alsaedi, J. Mol. Liq. 220, 642 (2016).

5. M.I. Khan, M. Waqas, T. Hayat, A. Alsaedi, J. Colloid Interface Sci. 498, 85 (2017).

6. T. Hayat, M.I. Khan, M. Waqas, A. Alsaedi, T. Yasmeen, Chin. J. Chem. Eng. 25, 257 (2017).

7. K. Vajravelu, J.R. Cannon, J. Leto, R. Semmoum, S. Nathan, M. Draper, D. Hammock, J. Math. Anal. Appl. 277, 609 (2003).

8. R. Nandkeolyar, S.S. Motsa, P. Sibanda, J. Nanotechnol. Eng. Med. 4, 041002 (2013).

9. S. Shaw, G. Mahanta, P. Sibanda, Alex. Eng. J. 55, 1295 (2016).

10. S.A. Shehzad, F.M. Abbasi, T. Hayat, A. Alsaedi, J. Mol. Liq. 224, 274 (2016).

11. F.M. Abbasi, S.A. Shehzad, J. Mol. Liq. 220, 848 (2016).

12. T. Hayat, S. Qayyum, S.A. Shehzad, A. Alsaedi, J. Mol. Liq. 230, 641 (2017).

13. A. Sharma, V.V. Tyagi, C.R. Chen, D. Buddhi, Renew. Sustain. Energy Rev. 13, 318 (2009). 
14. S.U.S. Choi, J.A. Eastman, Enhancing thermal conductivity of fluids with nanoparticles, in The Proceedings of the 1995 ASME International Mechanical Engineering Congress and Exposition, San Francisco, USA, FED 231/MD, 66 (ASME, 1995) pp. 99-105.

15. H. Masuda, A. Ebata, K. Teramae, N. Hishinuma, Netsu Bussei 7, 227 (1993).

16. B. Jalilpour, S. Jafarmadar, D.D. Ganji, A.B. Shotorban, H. Taghavifar, J. Mol. Liq. 195, 194 (2014).

17. M. Farooq, M.I. Khan, M. Waqas, T. Hayat, A. Alsaedi, M.I. Khan, J. Mol. Liq. 221, 1097 (2016).

18. T. Hayat, M. Waqas, M.I. Khan, A. Alsaedi, Int. J. Heat Mass Transfer 102, 1123 (2016).

19. M.J. Babu, N. Sandeep, J. Mol. Liq. 222, 1003 (2016).

20. A. Alsaedi, M.I. Khan, M. Farooq, N. Gull, T. Hayat, Adv. Powder Tech. 28, 288 (2017).

21. A. Abdollahi, M.R. Salimpour, Eur. Phys. J. Plus 131, 414 (2016).

22. T. Hayat, M.I. Khan, M. Waqas, A. Alsaedi, J.Mol. Liq. 231, 126 (2017).

23. S.S. Giri, K. Das, P.K. Kundu, Eur. Phys. J. Plus 132, 101 (2017).

24. S. Farooq, T. Hayat, A. Alsaedi, B. Ahmad, Int. J. Heat Mass Transfer 112, 521 (2017).

25. W.A. Khan, M. Irfan, M. Khan, A.S. Alshomrani, A.K. Alzahrani, M.S. Alghamdi, J. Mol. Liq. 234, 201 (2017).

26. A. Pantokratoras, T. Fang, Phys. Scr. 87, 015703 (2012).

27. R. Cortell, J. King Saud Univ. Sci. 26, 161 (2014).

28. Masood Khan, W.A. Khan, A.S. Alshomrani, Int. J. Heat Mass Transfer 101, 570 (2016).

29. T. Hayat, S. Qayyum, A. Alsaedi, B. Ahmad, Int. J. Heat Mass Transfer 110, 506 (2017)

30. B. Mahanthesh, B.J. Gireesha, G.T. Thammanna, S.A. Shehzad, F.M. Abbasi, R.S.R. Gorla, Alex. Eng. J. (2017) DOI: 10.1016/j.aej.2017.03.037.

31. T. Hayat, M.I. Khan, M. Farooq, T. Yasmeen, A. Alsaedi, J. Mol. Liq. 224, 786 (2016).

32. T. Hayat, M. Tamoor, M.I. Khan, A. Alsaedi, Results Phys. 6, 1031 (2016).

33. M.I. Khan, M. Tamoor, T. Hayat, A. Alsaedi, Results Phys. 7, 1207 (2017).

34. T. Hayat, M.I. Khan, M. Waqas, A. Alsaedi, Results Phys. 7, 256 (2017).

35. T. Hayat, G. Bashir, M. Waqas, A. Alsaedi, J. Mol. Liq. 223, 836 (2016).

36. T. Hayat, M. Waqas, S.A. Shehzad, A. Alsaedi, J. Mol. Liq. 222, 181 (2016). 